\title{
COMPLETELY CYCLIC INJECTIVE SEMILATTICES
}

\author{
C. S. JOHNSON, JR. AND F. R. MCMORRIS ${ }^{1}$
}

\begin{abstract}
We characterize semilattices $S$ with identity for which every cyclic $S$-system is injective. We note that this condition, unlike the $R$-module case, is not equivalent to the condition that every $S$-system is injective.
\end{abstract}

1. Introduction. A (right) $S$-system is a set $M$ acted on by a semigroup $S$ such that $m\left(s_{1} s_{2}\right)=\left(m s_{1}\right) s_{2}$ for all $m \in M$ and all $s_{1}, s_{2} \in S$. We shall call a semigroup $S$ completely injective if every $S$-system is injective and completely cyclic injective if every cyclic $S$-system is injective. In [4] B. Osofsky showed that these two notions coincide when they are formulated for $R$ modules. In the present paper we characterize completely cyclic injective semilattices and then use a characterization of completely injective semilattices due to Feller and Gantos [2] to show that these notions fail to coincide for $S$-systems. Finally, we discuss a generalization of our characterization suggested by the principal result of [2].

2. Completely cyclic injective semilattices. If $R$ and $M$ are $S$-systems, a map $\phi: R \rightarrow M$ is an $S$-homomorphism when $\phi(r) s=\phi(r s)$ for all $r \in R$ and all $s \in S$. An $S$-system $M$ is injective when every $S$-homomorphism from an $S$-subsystem $P$ of an $S$-system $R$ into $M$ has an extension to all of $R$. A semigroup $S$ with identity is completely injective when every unitary $S$-system is injective. An $S$-system $M$ is cyclic when there exists an $m \in M$ such that $M=m S$. Finally, we define a semigroup $S$ to be completely cyclic injective when every cyclic $S$-system is injective.

Throughout the remainder of this section $S$ will denote a semilattice with identity element 1 . If $\sim$ is a semilattice congruence on $S$ it is clear that $S / \sim$ is an $S$-system with $[x] s=[x s]$ and that it is cyclic. Thus it can be shown that every semilattice homomorphic image of $S$ is a cyclic $S$-system.

Lemma. Every cyclic S-system is isomorphic (as an $S$-system) to a semilattice homomorphic image of $S$.

Received by the editors January 10, 1972 and, in revised form, April 26, 1972.

AMS (MOS) subject claissifications (1970). Primary 20M99; Secondary 16A52.

Key words and phrases. $S$-systems, injective cyclic $S$-systems.

${ }^{1}$ Research of second author supported in part by a postdoctoral fellowship in the Biomathematics Program at North Carolina State University under PHS Grant \#GM-678 from NIGMS. Research of first author supported in part by a research grant from the Faculty Research Committee of Bowling Green State University.

(c) American Mathematical Society 1973 
Proof. Let $m S$ be a cyclic $S$-system. Define a relation $\sim$ on $S$ by $x \sim y$ if and only if $m x=m y$. It is easy to see that $\sim$ is a semilattice congruence on $S$ and that the map $m x \mapsto[x]$ is an $S$-isomorphism between $m S$ and $S / \sim$.

Lemma. A semilattice homomorphic image $T$ of $S$ is injective (in the category of $S$-systems) if and only if it is a complete lattice satisfying the following distributive laiv.

$$
s \wedge \bigvee A=\bigvee(s \wedge a \mid a \in A) \quad \text { for all } s \in T \text { and all } A \subseteq T \text {. }
$$

Proof. This follows from Corollary 4 of [3].

DEfINITION. A chain of diamonds is a lattice in which $a, b, c$ mutually distinct, $a \leq b$, and $b a$ imply that either $c<a$ and $c<b$, or $a<c$ and $b<c$. (We shall write $a \mid b$ when $a \pm b$ and $b \leq a$.)

If $I$ is an ideal of the semilattice $S$ we recall that the Rees factor semigroup $S / I$ is a semilattice homomorphic image of $S$ in which the elements of $I$ are identified as the zero of $S / I$. This is used repeatedly in the following proof along with the fact that every semilattice homomorphic image of $S$ is a cyclic $S$-system and hence a distributive lattice whenever it is injective.

THEOREM. S is completely cyclic injective if and only if it is a complete (as a lattice) chain of diamonds.

Proof. Suppose that every cyclic $S$-system is injective. $S$ itself is then injective and hence a complete lattice. We must show that it is a chain of diamonds. We will use $N_{5}$ to denote the five element nonmodular lattice and $M_{5}$ to denote the remaining five element nondistributive lattice.

Let us suppose that $a, b, c \in S$ are mutually distinct and that $a \mid b$. We first assume that $c \mid a$ and $c \mid b$ and we consider the three cases determined by the cardinality of $A=\{a \vee b, a \vee c, b \vee c\}$. If $|A|=1$, we identify the elements of the ideal $[0, a) \cup[0, b) \cup[0, c)$ to get a homomorphic image of $S$ which has as a sublattice $\{0, a, b, c, a \vee b \vee c\} \cong M_{5}$, a contradiction of distributivity. If $|A|=2$, we may suppose that $b \vee c<a \vee b=a \vee c$ and identify the elements of $[0, a) \cup[0, b) \cup[0, c)$ to get a homomorphic image which has as a sublattice $\{0, a, b, b \vee c, a \vee b\} \cong N_{5}$, again a contradiction of distributivity. If $|A|=3$, we consider subcases determined by the number of comparabilities in $A$. If there are none (i.e., if the elements of $A$ are mutually noncomparable) we identify the elements of $[0, a \vee b) \cup[0, a \vee c) \cup[0, b \vee c)$ to get a homomorphic image having as a sublattice $\{0, a \vee b, a \vee c, b \vee c$, $a \vee b \vee c\} \cong M_{5}$, a contradiction. Exactly one comparability in $A$ is not possible since, for example, $a \vee b \leqq a \vee c$ implies that $b \leqq a \vee c$, which implies that $b \vee c \leqq a \vee c$. The only way that exactly two comparabilities can exist without transitivity or the argument of the preceding sentence producing a 
third is to have one element of $A$ larger than the other two, say $a \mathrm{~V} b$, $b \vee c<a \vee c$. In this case we get a contradiction by identifying the elements of $[0, a) \cup[0, c)$ to get a homomorphic image having as a sublattice $\{0, a, c$, $b \vee c, a \vee c\} \cong N_{5}$. Finally, three comparabilities in $A$ are not possible since, for example, $a \vee b \leqq b \vee c \leqq a \vee c$ implies that $a \vee c \leqq b \vee c$, which implies that $a \vee c=b \vee c$. Thus we have eliminated the possibility that $c \mid a$ and $c \mid b$.

If $c<b$ we have either $a=a \vee c$ or $a \vee c=a \vee b$. This is true since we have $a \leqq a \vee c \leqq a \vee b$, and if $a<a \vee c<a \vee b$ we can identify the elements of $[0, a) \cup$ $[0, b)$ to get a homomorphic image having as a sublattice $\{0, b, a, a \vee c$, $a \vee b\} \cong N_{5}$. We can eliminate the possibility that $a \vee c=a \vee b$ by considering that in such a case the elements of $[0, a) \cup[0, c)$ could be identified to give a homomorphic image having as a sublattice $\{0, a, c, b, a \vee b\} \cong N_{5}$. Thus if $c<b$ we must have $a=a \vee c$ and hence $c<a$. Finally, suppose that $b<c$. By the preceding argument, $a \mid c$ would give $b<a$, a contradiction, so either $a<c$ or $c<a$. Since $c<a$ would give $b<a$ by transititivity, we must have $a<c$. This completes the proof that $S$ is a chain of diamonds.

To prove the converse, let us suppose that $S$ is a complete chain of diamonds. We must show that every semilattice homomorphic image of $S$ is a complete lattice satisfying (1). Let $f: S \rightarrow T$ be a semilattice epimorphism. Since $f$ is isotone it is clear that if $T$ is a lattice it must be a chain of diamonds. To show that $T$ is a complete lattice it will suffice to show that arbitrary joins exist in $T$. Let $\left\{f\left(s_{\alpha}\right)\right\}$ be a subset of $T$. If $\left\{f\left(s_{\alpha}\right)\right\}$ has a maximum element then it clearly has a join so let us suppose that it fails to have a maximum element. Let $s=\bigvee s_{\alpha}$. Clearly $f\left(s_{\alpha}\right) \leqq f(s)$ for all $\alpha$. To show that $f(s)$ is the desired join we suppose that $f\left(s_{\alpha}\right) \leqq f(u)$ for all $\alpha$. We wish to show that $f(x) \leqq f(u)$. There cannot exist an $s_{\beta}$ such that $u \leqq s_{\beta}$, for then we would have $f(u) \leqq f\left(s_{\beta}\right) \leqq f(u)$ and $\left\{f\left(s_{\alpha}\right)\right\}$ would have a maximum element. Suppose there exists an $s_{\beta}$ such that $u \mid s_{\beta}$. Then if $\alpha \neq \beta$, either $s_{\alpha} \leqq u \wedge s_{\beta}$ or $u \vee s_{\beta} \leqq s_{\alpha}$ since $S$ is a chain of diamonds. The second of these would give $u \leqq s_{\alpha}$, a possibility we have eliminated. Thus $s_{\alpha} \leqq u \wedge s_{\beta}$ for all $\alpha \neq \beta$ and we have $f\left(s_{\alpha}\right) \leqq f\left(u \wedge s_{\beta}\right)=f(u) \wedge f\left(s_{\beta}\right) \leqq f\left(s_{\beta}\right)$ for all $\alpha \neq \beta$, giving $\left\{f\left(s_{\alpha}\right)\right\}$ a maximum element again. The only remaining possibility is that $s_{\alpha} \leqq u$ for all $\alpha$ and thus $s=\bigvee s_{\alpha} \leqq u$, giving $f(s) \leqq f(u)$. Thus $T$ is a complete lattice and it only remains to show that a complete chain of diamonds satisfies (1). We must show that $a \wedge \bigvee b_{\alpha} \leqq \bigvee\left(a \wedge b_{\alpha}\right)$ and to do this we consider three cases. First, if $a \leqq b_{\beta}$ for some $\beta$ then $a \wedge \bigvee b_{\alpha}=a \leqq \bigvee\left(a \wedge b_{\alpha}\right)$. Second, if $a \mid b_{\beta}$ for some $b_{\beta}$ then for each $\alpha \neq \beta$ either $b_{\alpha}<b_{\beta}$ or $b_{\beta}<b_{\alpha}$. If $b_{\beta}<b_{\alpha}$ for some $\alpha \neq \beta$, then $a \mid b_{\beta}$ gives $a<b_{\alpha}$ and we are back in the first case. If $b_{\alpha}<b_{\beta}$ for all $\alpha \neq \beta$, then $\bigvee b_{\alpha}=b_{\beta}$ and we have $a \wedge \bigvee b_{\alpha}=a \wedge b_{\beta} \leqq \bigvee\left(a \wedge b_{\alpha}\right)$. Finally, if $b_{\alpha} \leqq a$ for all $\alpha$, then $\bigvee b_{\alpha} \leqq a$ and we have $a \wedge \bigvee b_{\alpha}=\bigvee b_{\alpha}=$ $\bigvee\left(a \wedge b_{\alpha}\right)$. Thus a complete chain of diamonds satisfies (1) and we have shown that every cyclic $S$-system is injective. 
Theorem 2.9 of [2] says that a semigroup with central idempotents is completely injective if and only if it is a semilattice of groups whose idempotents form a complete dually well-ordered chain. Thus completely injective semilattices may be characterized as complete dually wellordered chains and it is clear, in light of our theorem, that a completely cyclic injective semilattice need not be completely injective.

3. Semigroups with central idempotents. In light of Feller and Gantos' result one might conjecture that the completely cyclic injective semigroups with central idempotents are the semilattices of groups whose idempotents form a complete chain of diamonds. We show that this is not the case by offering a counterexample.

First we recall that an $S$-system $M$ is weakly injective if for any right ideal $I$ of $S$ and $S$-homomorphism $\phi: I \rightarrow M$ there exists an $m \in M$ such that $\phi(x)=m x$ for all $x \in I$. It is shown in [1, p. 264] that an injective $S$ system is weakly injective.

Let $G=\{e, x\}$ be the cyclic group of order two with identity $e$ and let $S=G \cup\{f\} \cup\{0\} \cup\{1\}$ where 1 and 0 are the identity and zero of $S$ and $f^{2}=f$. Define $f G=0=G f$. Then $S$ is a semilattice of groups whose idempotents are a diamond. Consider the ideal $I=G \cup\{f\} \cup\{0\}$ and define $\phi: I \rightarrow S$ by $\phi(x)=e, \phi(e)=x, \phi(f)=f$, and $\phi(0)=0$. Then $\phi$ is an $S$ homomorphism but is not realized by any element of $S$. Hence $S$ is not completely cyclic injective since $S$ itself is not even weakly injective.

\section{REFERENCES}

1. P. Berthiaume, The injective envelope of $S$-sets, Canad. Math. Bull. 10 (1967), 261-273. MR 35 \#4321.

2. E. H. Feller and R. L. Gantos, Completely injective semigroups with central idempotents, Glasgow Math. J. 10 (1969), 16-20. MR 40 \#256.

3. C. S. Johnson, Jr. and F. R. McMorris, Injective hulls of certain S-systems over a semilattice, Proc. Amer. Math. Soc. 32 (1972), 371-375.

4. B. L. Osofsky, Rings all of whose finitely generated modules are injective, Pacific $\mathrm{J}$. Math. 14 (1964), 645-650. MR 28 \#5090.

Department of Mathematics, Bowling Green State University, Bowling GREEN, OHIO 43403

Biomathematics Program, North Carolina State University, Raleigh, North CAROLINA 27607 